\title{
Eye Salvage with Combination of Intravitreal and Intracameral Melphalan Injection for Recurrent Retinoblastoma with Anterior Chamber Involvement: Report of a Case
}

\author{
Nathalie Cassoux ${ }^{a}$, b, e Isabelle Aerts ${ }^{c}$ Livia Lumbroso-Le Rouic ${ }^{a}$ \\ Paul Freneaux $^{d}$ Laurence Desjardins ${ }^{a}$ \\ Departments of a Ocular Oncology, ${ }^{\mathrm{b}}$ Surgical Oncology, ${ }^{\mathrm{C} P e d i a t r i c}$ Oncology and ${ }^{\mathrm{d}}$ Pathology, Institut Curie, and \\ eUniversity René Descartes Paris V, Paris, France
}

\section{Keywords}

Intravitreal injection · Retinoblastoma · Treatment ·

Melphalan

\begin{abstract}
Aims: Herein we report successful eye salvage following combined intravitreal and intracameral administration of melphalan for anterior chamber recurrence of retinoblastoma. Methods: A 12-month-old boy was referred with a sporadic bilateral retinoblastoma staged as group $D$ and $V$ in the right eye and group $E$ and $V$ in the left eye. Treatment initially involved 6 cycles of 3 drugs followed by focal treatments. The left eye was enucleated. Three months after the end of systemic chemotherapy, multiple recurrent lesions were noted in the ciliary body and the anterior chamber. A series of intravitreal (IVT) and intracameral (IC) injections of melphalan combined with a 7th cycle of systemic chemotherapy was performed. The therapeutic schedule alternated: week $A$ an IVT of melphalan $(30 \mu \mathrm{g} / 0.1 \mathrm{~mL})$ for a total of 4 versus week B IC injections of melphalan ( $15 \mu \mathrm{g} / 0.05 \mathrm{~mL}) 4 \mathrm{IC}$ in total. Each injection was preceded by an anterior chamber. Cryotherapy
\end{abstract}

๑) 2016 S. Karger AG, Basel

E-Mail karger@karger.com www.karger.com/oop to the injection punctures was systematically performed. $\boldsymbol{R e}$ sults: Rapidly, iris and ciliary body nodules regressed completely based on clinical and ultrasound biomicroscopic examinations. After 3 years of follow-up, the patient has shown no evidence of recurrence. Conclusion: In our patient, combined IVT and IC administration of melphalan proved highly efficacious in eradicating the ciliary body and anterior chamber disease and salvage of his one remaining functional eye.

(c) 2016 S. Karger AG, Basel

\section{Introduction}

Tumor seeding of the anterior chamber of the eye is one of the most significant therapeutic challenges encountered in relapsing retinoblastoma and often necessitates enucleation or radiation therapy of the ocular globe [1]. Herein we report successful eye salvage following combined intravitreal (IVT) and intracameral (IC) administration of melphalan for anterior chamber recurrence of retinoblastoma in a child with previously treated sporadic bilateral retinoblastoma. 


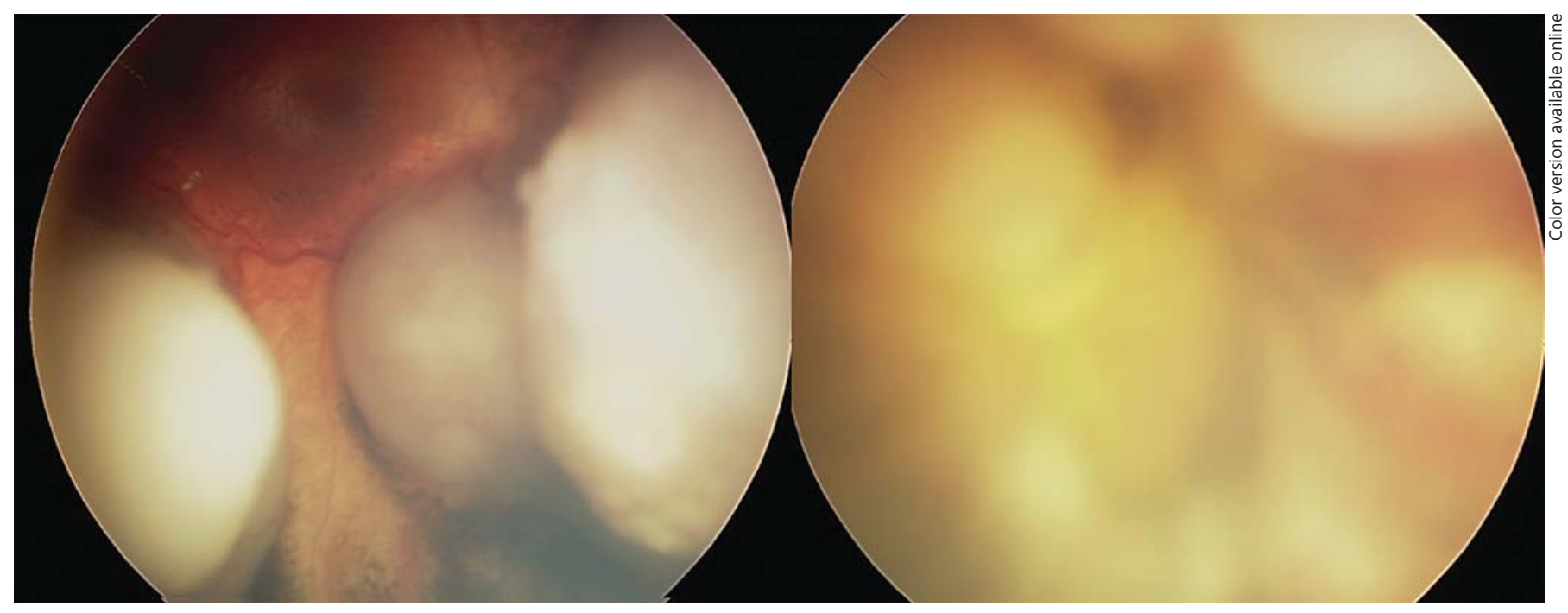

Fig. 1. Fundus photographs at onset showing bilateral advanced retinoblastoma.

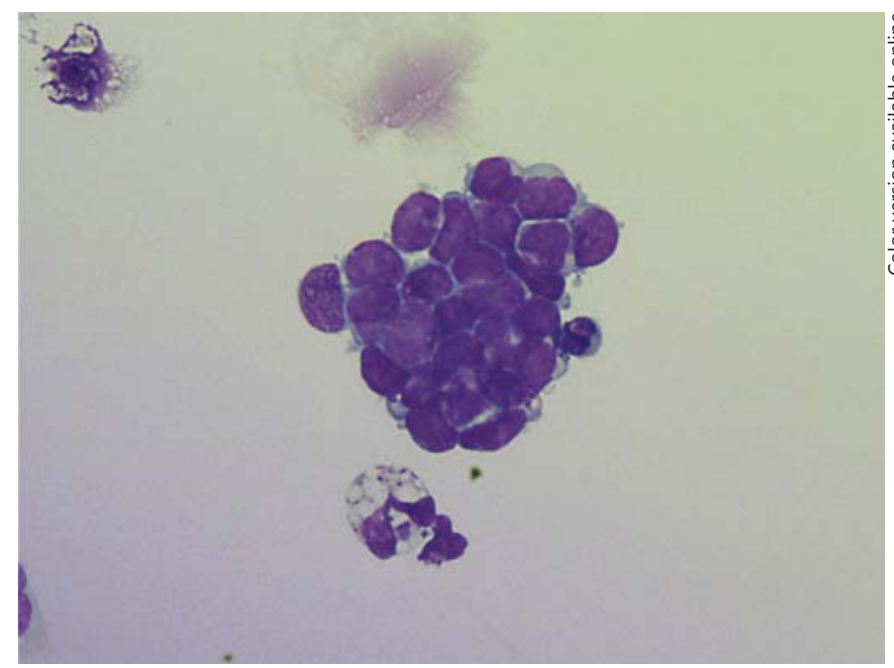

Fig. 2. Tumor cells disclosed on the cytological examination of the first anterior chamber tap.

\section{Report of Case}

A 12-month-old boy was referred to our institution for the management of sporadic bilateral retinoblastoma in May 2013. On examination, the patient was staged as group D and V (diffuse vitreous seeding) for the right eye and advanced group $\mathrm{E}$ and $\mathrm{V}$ (retinal detachment and neovascular glaucoma) for the left eye (Fig. 1). Treatment initially involved 6 cycles of combined vincristine, carboplatine, and etoposide followed by a second cycle of transpupillary thermotherapy (4 sessions for the right eye). The left eye was enucleated because of persistent vitreous hemorrhage and neovascular glaucoma. Following the termination of systemic chemotherapy, tumor recurrences in the retina at 1 and at 2 months were

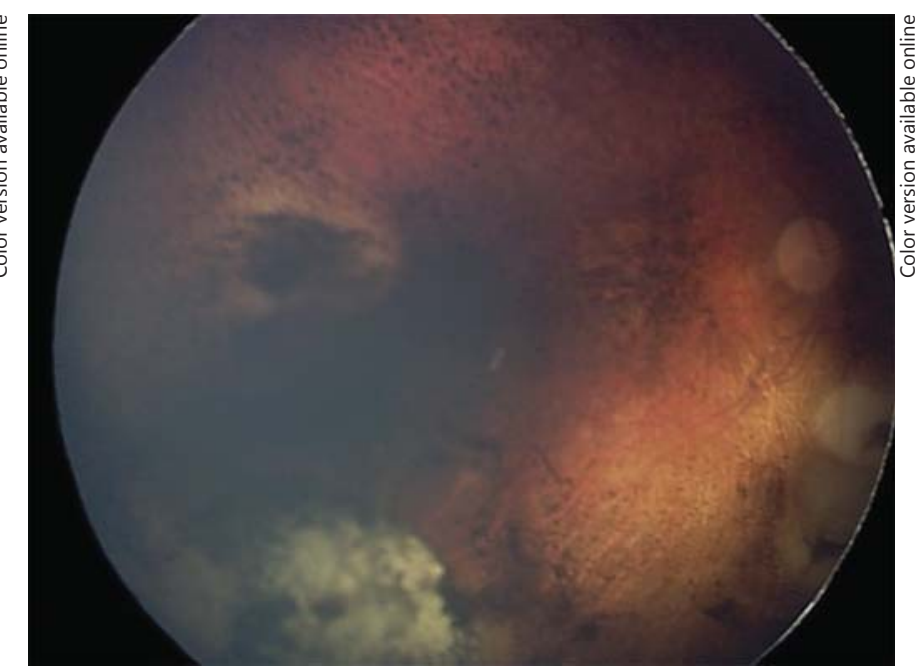

Fig. 3. Fundus photograph of the remaining right eye showing tumor scar and choroid and retina atrophy.

treated with cryotherapy. Three months after the end of systemic chemotherapy, multiple recurrent lesions were noted in the ciliary body and the anterior chamber, as evidenced by seeding and three nodules in the iris. A somatic and germline mutation on Rb1 gene exon 4 (c.471_475del/p.Leu158Cysfs*11) was identified. In order to avoid external beam radiotherapy, an intra-arterial injection of melphalan was administered but failed to clear the anterior chamber. It was then decided to use a series of IVT and IC injections of melphalan combined with a 7th cycle of systemic chemotherapy, as specified above, in an effort to diminish the risk of distant metastasis. The therapeutic schedule alternated: week A, an IVT injection of melphalan $(30 \mu \mathrm{g} / 0.1 \mathrm{~mL})$ for a total of 4 injections versus week B, IC injections of melphalan $(15 \mu \mathrm{g} / 0.05 \mathrm{~mL}), 4$ injections 
in total. Each injection was preceded by an anterior chamber tap in order to circumvent an elevation of intraocular pressure, to avoid any reflux, and for the potential detection of tumor cells. Cryotherapy to the injection punctures (the anterior chamber tap and IVT injection site) was systematically performed. The initial anterior chamber tap revealed retinoblastoma tumor cells (Fig. 2); however, rapidly after the first round of combination IC/IVT therapy, the anterior chamber was clear of tumor cells. The iris and ciliary body nodules regressed completely based on clinical and ultrasound biomicroscopic examinations. After 3 years of followup, the patient has shown no evidence of recurrence. Examination of the right eye revealed moderate synechiae of the iris but the lens has remained clear. The fundus manifested atrophic scarring and a choroidal and retinal vasculature toxicity (Fig. 3). Despite this, the child has retained a useful vision.

\section{Discussion}

Recurrence of retinoblastoma in the anterior chamber is a well-recognized cause of conservative treatment failure of this neoplasm [2]. Enucleation of the globe is often necessary, and if anterior chamber involvement is confirmed histopathologically, adjuvant chemotherapy is usually recommended [3]. This type of approach is difficult to propose for patients with only one functional eye. Alternatively, external beam radiotherapy may be considered; however, one must bear in mind not only treatment failure but also the associated increased risk for the development of a second cancer in the irradiated field, especially for patients with a constitutional mutation $[4,5]$.

Intra-arterial chemotherapy has been introduced in recent years as a form of salvage therapy in retinoblastoma [6]. However, this modality has not appeared efficacious for anterior chamber seeding based on the studies by Pavlidou et al. [7]. In our case, the initial intra-arterial infusion with melphalan also appeared unable to clear the ciliary body and the anterior chamber [7]. Recently, IVT injection of melphalan or topotecan has also shown promise as a means of eradicating vitreous seeding of retinoblastoma with acceptable side effects [8]. A recent paper demonstrated that after an IVT injection of melphalan in a rabbit eye, the concentrations of the drug in the vitreous and anterior chamber were, respectively, 7.8 and $0.024 \mu \mathrm{g} / \mathrm{mL}$, with a rapid clearance of the drug in the anterior chamber [9]. This pharmacokinetic study showed that in case of anterior chamber involvement, IVT injection alone is probably not sufficient. A salvage technique using anterior chamber and IVT injection of melphalan during the same and unique procedure was described by Munier et al. [2] in 2015. This technique delivers the drug in the two compartments after an aspiration of an equiv- alent volume of both aqueous humor and vitreous. Aspiration and injection was done via a long needle introduced across the cornea and going through the iris and ciliary body to reach the vitreous cavity. In our technique, we removed only aqueous humor without aspiration of the vitreous and without touching the iris and ciliary body [2]. In contrast to the cases described in this abstract, our patient had an anterior chamber involvement and an iris and ciliary body infiltration. It will be dangerous to go through ocular structures invaded by tumor cells with a needle and remove this needle outside the eye. These local or locoregional treatments must be used after careful selection of the patients. A recent meta-analysis of intra-arterial chemotherapy for retinoblastoma has suggested an increased risk of metastasis in patients with advanced retinoblastoma treated with this modality [10]. Nonetheless, to date, there are no reported cases of orbital recurrence after IVT injection of melphalan. Anyway, caution must be exercised when conspicuous vitreous tumor seeding is present [11].

\section{Conclusion}

In our patient with recurrent retinoblastoma involving the anterior chamber, combined IVT and IC administration of melphalan proved highly efficacious in eradicating the ciliary body and anterior chamber disease and salvage of his one remaining functional eye. Studies of additional, similarly affected patients appear indicated.

\section{Acknowledgements}

Thanks to Prof. Raymond Barnhill, who kindly reviewed the paper.

\section{Statement of Ethics}

Parents gave consent for publication of their child's medical history and fundus pictures. Ethics committee approval is not necessary in France.

\section{Disclosure Statement}

The authors have no conflict of interest. 


\section{References}

1 Shields CL, Lally SE, Manjandavida FP, Leahey AM, Shields JA: Diffuse anterior retinoblastoma with globe salvage and visual preservation in 3 consecutive cases. Ophthalmology 2016;123:378-384.

2 Munier FL, Gaillard MC, Decembrini S, et al: Aqueous seeding: fall of the ultimate intraocular retinoblastoma sanctuary by a new in situ technique. Invest Ophthalmol Vis Sci 2015; 56:abstract 1663.

3 Sastre X, Chantada GL, Doz F, Wilson MW, de Davila MT, et al: Proceedings of the consensus meetings from the International Retinoblastoma Staging Working Group on the pathology guidelines for the examination of enucleated eyes and evaluation of prognostic risk factors in retinoblastoma. Arch Pathol Lab Med 2009;133:1199-1202.
4 Aerts I, Pacquement H, Doz F, Mosseri V, Desjardins L, et al: Outcome of second malignancies after retinoblastoma: a retrospective analysis of 25 patients treated at the Institut Curie. Eur J Cancer 2004;40:1522-1529.

5 Dunkel IJ, Gerald WL, Rosenfield NS, Strong EW, Abramson DH, et al: Outcome of patients with a history of bilateral retinoblastoma treated for a second malignancy: the Memorial Sloan-Kettering experience. Med Pediatr Oncol 1998;30:59-62.

6 Abramson DH, Daniels AB, Marr BP, Francis $\mathrm{JH}$, Brodie SE, et al: Intra-arterial chemotherapy (ophthalmic artery chemosurgery) for group D retinoblastoma. PLoS One 2016; 11:e0146582.

7 Pavlidou E, Burris C, Thaung C, Scheimberg I, Kingston J, et al: Anterior segment seeding in eyes with retinoblastoma failing to respond to intraophthalmic artery chemotherapy. JAMA Ophthalmol 2015;133:1455-1458.
8 Francis JH, Marr BP, Brodie SE, Abramson DH: Anterior ocular toxicity of intravitreous melphalan for retinoblastoma. JAMA Ophthalmol 2015;133:1459-1463.

9 Buitrago E, Winter U, Williams G, Asprea M, Chantada G, et al: Pharmacokinetics of melphalan after intravitreal injection in a rabbit model. J Ocul Pharmacol Ther 2016;32:230235

10 Yousef YA, Soliman SE, Astudillo PP, Durairaj $P$, Dimaras $H$, et al: Intra-arterial chemotherapy for retinoblastoma: a systematic review. JAMA Ophthalmol 2016;134:584-591.

11 Munier FL, Soliman S, Moulin AP, Gaillard MC, Balmer A, et al: Profiling safety of intravitreal injections for retinoblastoma using an anti-reflux procedure and sterilisation of the needle track. Br J Ophthalmol 2012;96:10841087. 